DOI: 10.3201/eid1704.102027

Suggested citation for this article: Chang H-W, Egberink HF, Rottier PJM. Sequence analysis of feline coronaviruses and the circulating virulent/avirulent theory [letter]. Emerg Infect Dis. 2011 Apr; [Epub ahead of print]

\title{
Sequence Analysis of Feline Coronaviruses and the Circulating Virulent/Avirulent Theory
}

To the Editor: Feline coronaviruses (FCoVs) occur as 2 pathotypes, feline infectious peritonitis virus (FIPV) and feline enteric coronavirus (FECV). FECV is common in cats, causing mild transient enteritis in kittens, but is asymptomatic in adult cats. In contrast, FIPV occurs sporadically but is lethal. It replicates in monocytes and macrophages and rapidly disseminates throughout the body causing systemic immunopathologic disease (1-4).

The relationship between FECV and FIPV has become a matter of debate. Genetic and animal experimental evidence indicates that FIPV arises by mutation from FECV in the intestinal tract of a persistently infected cat; the virus thereby acquires the monocyte or macrophage tropism that enables it to spread systemically and cause FIP $(5-7,8)$. According to another view, the 2 pathotypes circulate independently in the field. This circulating virulent/avirulent FCoV theory recently was advocated by Brown et al. (9). Their conclusion was based on sequence analyses of parts of the viral genome including the matrix (M) gene, phylogenetic analysis of which revealed reciprocal monophyly of the sequences obtained from FIP cases versus those of asymptomatic FECV-infected animals. In addition, the authors suggested 5 aa residues in the M protein to represent potential diagnostic markers for distinguishing virulent FIPV from avirulent FECV (9).

To try to verify the findings of Brown et al. (9), we determined and analyzed M genes from $43 \mathrm{FCoV}$ genomes, 20 of which came from cats in single-cat households, and 23 from cattery animals. The latter group consisted of 10 asymptomatic healthy cats (FECV; test specimens: feces) and 13 dead cats with FIP confirmed through pathology (FIPV; test 
specimens: organs, ascites). These animals came from 8 catteries. FECV and FIPV cases were found in 7 (designated $A$ to $\mathrm{G}$ ); the remaining cattery $(\mathrm{H})$ provided 2 cats with FIP. The genomes from individual live cats were from 15 FIPV- and 5 FECV-infected animals.

Using specific primers (sense 5'-CGTCTCAATCAAGGCATATAATCCCGACGAAG3', antisense 5'-CAGTTGACGCGTTGTCCCTGTG-3'), we amplified the same 575-bp M gene fragment as studied by Brown et al. (9). GenBank accession numbers for the FCoV M gene sequences determined in this study are HQ738691-HQ738733. When compared by phylogenetic analysis, the nucleotide sequences of FIPV and FECV M genes distributed into paraphyletic patterns rather than in monophyletic clusters (Figure, panel A).

Thus, as we observed earlier for the $3 \mathrm{c}$ gene (10), $\mathrm{M}$ gene sequences generally clustered according to the cattery from where they originated, irrespective of their pathotype (e.g., FECV 586 and FIPVs 584 and 585 from cattery A; FECV 620 and FIPVs 615 and 622 from cattery G; FECV 10 and FIPV 8 from cattery F). Such a distribution pattern is consistent with the mutation theory, according to which FIPVs originate from FECVs and are thus closely related $(7,9)$. Exceptions in this picture were FIPV 9 in cattery F and FECVs 406 and 407 in cattery D, presumably caused by multiple FCoV lineages in these open catteries (an open cattery is one in which cats routinely move in and out, usually for breeding purposes).

We also examined the 5 aa sites in the M protein identified by Brown et al. (9) as being potentially diagnostic of FIP. An alignment of the relevant part of the polypeptide sequence, comprising the presumed signature residues at positions 108, 120, 138, 163 and 199, is shown in the Figure, panel B, for all FIPV and FECV genomes sequenced in this study. Within this sample collection, we observed complete sequence conservation at positions 108 and 199, virtually complete conservation ( 1 difference) at position 163. The 2 aa identities (Val and Ile) found at position 120 and 138 occurred with similar frequencies in FIPV and FECV (position 120: Ile in 16 of 36 (44\%) of FIPVs and in 6 of 14 (43\%) of FECVs; position 138: Ile in 29/36 or 81\% of FIPVs and in $12 / 14$ or $86 \%$ of FECVs). These observations do not indicate the slightest tendency of sequence segregation among the 2 pathotypes. In the alignment of the Figure, panel B, we also included $\mathrm{M}$ protein sequences translated from several $\mathrm{FCoV}$ genomes from the Americas, 7 FIPV, and 1 FECV (8). The comparison does not reveal peculiarities indicative of geographic 
segregation. Hence, our data do not confirm the diagnostic potential of the M protein sequence nor do they support the suggested role of the membrane protein in FIP pathogenesis (9).

Informative as it may be, comparative sequence analysis will eventually not be sufficient to answer the FECV/FIPV question. What will be needed is a reverse genetics system to generate and manipulate the FCoV genome as well as a cell culture system to propagate the viruses, both of which have thus far not been achieved.

\section{Acknowledgments}

We thank the veterinarians and cat owners in the Netherlands who provided FIP cases and fecal material for this study. We also thank Raoul de Groot for his constructive remarks on the manuscript.

Hui-Wen Chang, Herman F. Egberink, and Peter J.M. Rottier

Author affiliation: Utrecht University, Utrecht, The Netherlands

\section{References}

1. Addie D, Belák S, Boucraut-Baralon C, Egberink H, Frymus T, Gruffydd-Jones T, et al. Feline infectious peritonitis. ABCD guidelines on prevention and management. J Feline Med Surg. 2009;11:594-604. PubMed DOI: 10.1016/j.jfms.2009.05.008

2. de Groot RJ, Horzinek MC. Feline infectious peritonitis. In: The Coronaviridae. Siddell SG, editor. New York: Plenum Press; 1995. p. 293-309.

3. Haijema BJ, Rottier PJ, de Groot RJ. Feline coronaviruses: a tale of two-faced types. In: Thiel V, editor. Coronaviruses. Molecular and cellular biology. Norfolk (UK): Academic Press; 2007. p. 183-203.

4. Pedersen NC. A review of feline infectious peritonitis virus infection: 1963-2008. J Feline Med Surg. 2009;11:225-58. PubMed DOI: 10.1016/j.jfms.2008.09.008

5. Herrewegh AA, Vennema H, Horzinek MC, Rottier PJM, de Groot RJ. The molecular genetics of feline coronaviruses: comparative sequence analysis of the ORF $7 \mathrm{a} / 7 \mathrm{~b}$ transcription unit of different biotypes. Virology. 1995;212:622-31. PubMed

6. Poland AM, Vennema H, Foley JE, Pedersen NC. Two related strains of feline infectious peritonitis virus isolated from immunocompromised cats infected with a feline enteric coronavirus. J Clin Microbiol. 1996;34:3180-4. PubMed 
7. Vennema H, Poland A, Foley J, Pedersen NC. Feline infectious peritonitis viruses arise by mutation from endemic feline enteric coronaviruses. Virology. 1998;243:150-7. PubMed DOI: $\underline{10.1006 / \text { viro. } 1998.9045}$

9. Brown MA, Troyer JL, Pecon-Slattery J, Roelke ME, O’Brien SJ. Genetics and pathogenesis of feline infectious peritonitis virus. Emerg Infect Dis. 2009;15:1445-52. PubMed DOI: $\underline{10.3201 / \text { eid1509.081573 }}$

10. Chang HW, de Groot RJ, Egberink HF, Rottier PJ. Feline infectious peritonitis: insights into feline coronavirus pathobiogenesis and epidemiology based on genetic analysis of the viral $3 \mathrm{c}$ gene. $\mathrm{J}$ Gen Virol. 2010;91:415-20. PubMed DOI: 10.1099/vir.0.016485-0

8. Pedersen NC, Liu H, Dodd KA, Pesavento PA. Significance of coronavirus mutants in feces and diseased tissues of cats suffering from feline infectious peritonitis. Viruses. 2009;1:166-84. DOI: $\underline{10.3390 / \mathrm{v} 1020166}$

Address for correspondence: Peter J.M. Rottier, Virology Division, Department of Infectious Diseases \& Immunology, Veterinary Faculty, Utrecht University, Yalelaan 1, 3584 CL Utrecht, the Netherlands; email: p.rottier@uu.nl 


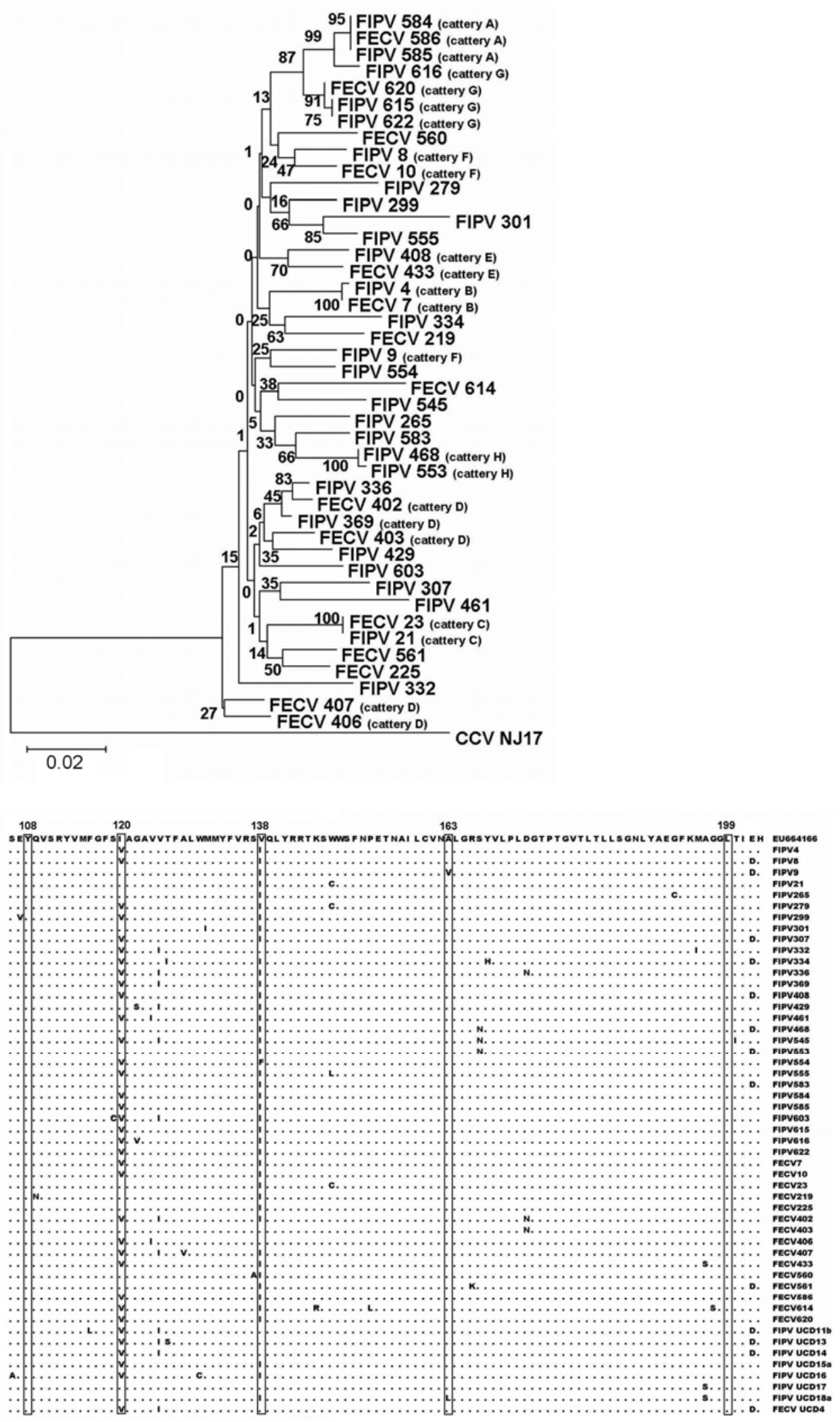

Figure. A) Phylogenetic relationships of feline coronaviruses (FCoVs) detected in feces of healthy cats and in organs/ascites of cats with feline infectious peritonitis. Alignment of the matrix (M) gene sequences 
was used to generate a rooted neighbor-joining tree with the $\mathrm{M}$ gene sequence of canine coronavirus strain NJ17 (Genbank accession no. AY704917) as outgroup. Bootstrap confidence values (percentages of 1,000 replicates) are indicated at the relevant branching points. Branch lengths are drawn to scale; scale bar indicates 0.02 nucleotide substitutions per site. Viruses detected in cattery animals are indicated by a cattery designation after the virus. identification B) Alignment of amino acid sequences of partial M proteins of the FCoVs from panel A, as compared with a feline infectious peritonitis virus (FIPV) reference sequence (top line) published by Brown et al. (9) (GenBank accession no. EU664166), and with 8 American FCoV sequences (bottom) published by Pedersen et al. (8). The 5 aa residues at positions $108,120,138,163$, and 199, suggested by Brown and others (9) as potential diagnostic sites, are boxed. 